Check for updates

Cite this: RSC Adv., 2018, 8, 31201

Received 9th August 2018

Accepted 28th August 2018

DOI: $10.1039 / c 8 r a 06691 d$

rsc.li/rsc-advances

\section{Synthesis of novel genistein amino acid derivatives and investigation on their interactions with bovine serum albumin by spectroscopy and molecular docking $\dagger$}

\author{
Xiaokang Long, \$ Yao-Fu Zeng, \$Y Yunmei Liu, Ying Liu, Tangluo Li, Lanqing Liao \\ and Yu Guo (DD*
}

Genistein amino acid derivatives $4 a-4 d$ were synthesized and evaluated for their cytotoxic activities against MCF-7, Hela, MGC-803 and HCT-116 cell lines by MTT assays in vitro. The results revealed that compounds $4 a-4 d$ showed better activity than the parent compound genistein. Particularly, compound $4 \mathrm{~b}$ displayed the most significant anticancer activity against MGC-803 with an $\mathrm{IC}_{50}$ value of $12.08 \mu \mathrm{M}$. In addition, the mechanisms of interaction between genistein, compounds $4 a-4 d$ and BSA were investigated via multispectroscopic techniques such as ultraviolet (UV) spectroscopy, fluorescence, circular dichroism (CD), and molecular docking under physiological conditions. The results suggested that endogenous fluorescence of BSA could be quenched by genistein and compounds $4 a-4 d$ via forming BSAcompound complex, which meant a static quenching mechanism was involved. The negative values of enthalpy $(\Delta H)$ and entropy $(\Delta S)$ indicated that interactions between BSA and the ligands were spontaneous, and hydrogen bonding and van der Waals interactions were involved in the BSAcompound complexion formation. The UV, synchronous and 3D fluorescence results revealed that the micro-environment of tryptophan and conformation of BSA were changed after binding to ligands. CD analysis demonstrated the variation in the secondary structure and that the $\alpha$-helix content of BSA decreased. Eventually, molecular docking was executed to forecast the binding forces and binding sites between BSA and compounds $4 \mathrm{a}-4 \mathrm{~d}$.

\section{Introduction}

Serum albumins, indispensable parts of the plasma, play vital roles in the absorption, distribution, metabolism, and excretion profiles of various endogenous and exogenous compounds. ${ }^{1}$ Consequently, exploring the interaction between drugs and serum albumins could provide messages for us to interpret the metabolism and transport mechanism of drugs and design new compounds with better biological activities and lower toxicities. Among serum albumins, bovine serum albumin (BSA) is often used as a model for studying the binding of drugs to serum albumin, because it is available and similar to human serum albumin in structure. ${ }^{2,3}$ BSA has three linearly arranged domains (I-III), and each domain consists of A and B subdomains. Besides, it contains two amino acid residues

Hunan Province Cooperative Innovation Center for Molecular Target New Drug Study Institute of Pharmacy and Pharmacology, University of South China, Hengyang, 421001, China. E-mail: guoyuhy@126.com

$\dagger$ Electronic supplementary information (ESI) available. See DOI: 10.1039/c8ra06691d

\$ These authors contributed equally to this work. (tryptophan 134 and tryptophan 212) with endogenous fluorescence. $^{4,5}$ (Fig. 1a).

Genistein, widely existing in soybean, possesses a broad range of pharmacological activities such as antitumor, antioxidant, antiseptic, anti-osteoporotic and so on.$^{6-9}$ However, its poor solubility and low bioavailability limited its potential in clinical treatment. Amino acids are the basic components of protein and participate in a variety of physiological activities in the body. The introduction of amino acids into drugs as amino acid prodrugs has become a popular strategy for scholars. A large number of experimental data showed that amino acid prodrugs can improve solubility, permeability as well as metabolic stability of the parent substances. ${ }^{\mathbf{1 0 - 1 2}}$ In recent years, the interactions between chlorinated, ${ }^{\mathbf{1 3}}$ alkylated and trifluoromethylated genisteins and BSA were reported by several groups. ${ }^{14}$ However, the studies on the structural affinity relationship between genistein amino acid derivatives and BSA have not yet been published.

Herein four genistein conjugates modified with amino acid 4a-4d (Fig. 1b) were synthesized and evaluated as anti-cancer agents. Besides, the mechanisms of interaction between genistein amino acid derivatives $\mathbf{4 a - 4 d}$ and BSA were explored 

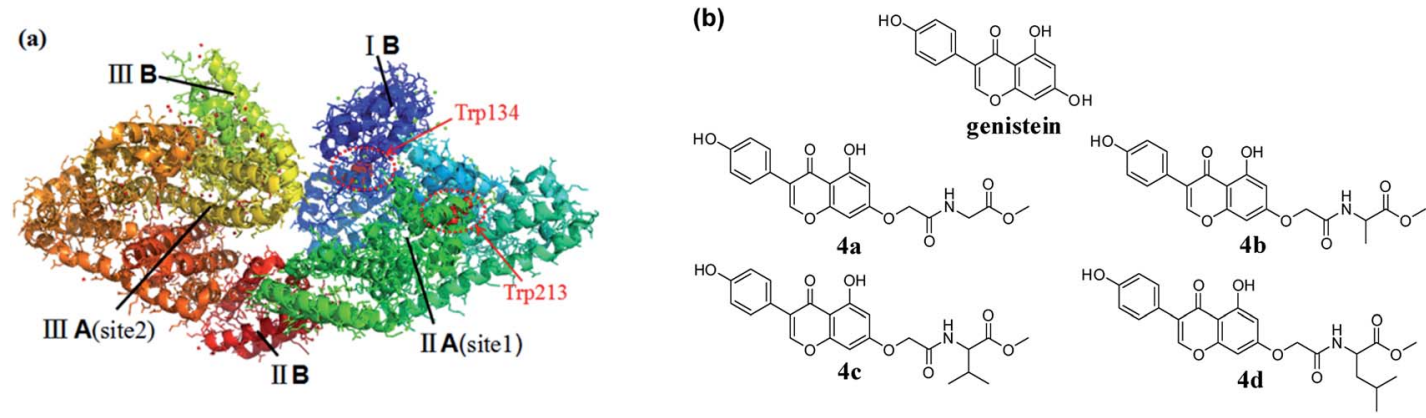

Fig. 1 (a) The crystal structure of BSA; (b) the chemical structures of genistein and $4 a-4 d$.<smiles>CCOC(=O)COc1cc(O)c2c(=O)c(-c3ccc(O)cc3)coc2c1</smiles><smiles>O=C(O)COc1cc(O)c2c(=O)c(-c3ccc(O)cc3)coc2c1</smiles>
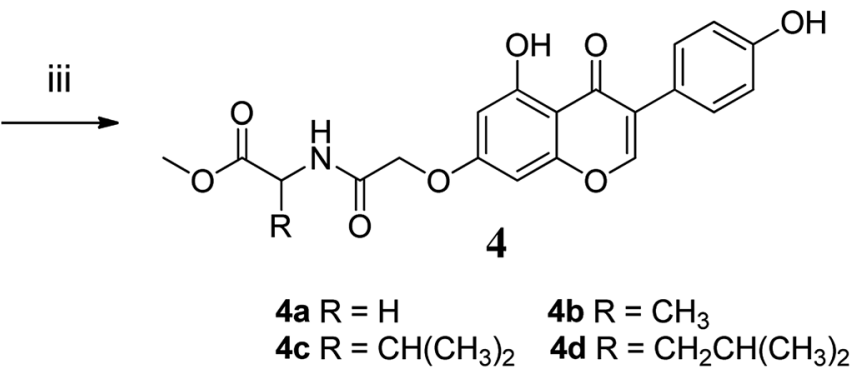

Fig. 2 Reagents and conditions: (i) $\mathrm{KOH}, \mathrm{DMF}, 60^{\circ} \mathrm{C}, 1 \mathrm{~h}$, then $\mathrm{BrCH}_{2} \mathrm{COOC}_{2} \mathrm{H}_{5}, 60^{\circ} \mathrm{C}, 5 \mathrm{~h}$; (ii) $\mathrm{CH}_{3} \mathrm{OH}, \mathrm{H}_{2} \mathrm{O}, \mathrm{KOH}\left(1 \mathrm{~mol} \mathrm{~L}{ }^{-1}\right), 60{ }^{\circ} \mathrm{C}, 0.5 \mathrm{~h}$, then $\mathrm{H}_{2} \mathrm{SO}_{4}\left(0.5 \mathrm{~mol} \mathrm{~L}^{-1}\right)$, at room temperature, $0.5 \mathrm{~h}$; (iii) EDCl, HOBT, DMF, $0{ }^{\circ} \mathrm{C}, 4 \mathrm{~h}$, then DIPEA, DMAP, amino acid methyl ester, r.t., $24 \mathrm{~h}$.

using multi-spectroscopy (UV, synchronous fluorescence, 3D fluorescence, CD spectroscopy) and molecular docking.

\section{Materials and methods}

\subsection{Reagent and apparatus}

Genistein $(\geq 97 \%)$ and BSA ( $\geq 99 \%)$ were obtained from Aladdin's Reagent Co., Ltd. (Shanghai, China). Tris(hydroxymethyl)

Table 1 Cytotoxic activities of genistein and its amino acid derivatives $4 a-4 d$

\begin{tabular}{lllll}
\hline & \multicolumn{4}{l}{ Cancer cell lines $\left(\mathrm{IC}_{50}, \mu \mathrm{M}\right)^{a}$} \\
\cline { 2 - 5 } Compounds & MCF-7 & Hela & MGC-803 & \multicolumn{1}{l}{ HCT-116 } \\
\hline 4a & $>100$ & $66.63 \pm 3.60$ & $24.35 \pm 1.27$ & $65.28 \pm 2.91$ \\
4b & $21.32 \pm 2.24$ & $90.27 \pm 0.47$ & $12.08 \pm 0.06$ & $>100$ \\
4c & $99.73 \pm 0.11$ & $82.71 \pm 0.15$ & $33.53 \pm 0.13$ & $42.64 \pm 0.15$ \\
4d & $47.00 \pm 3.53$ & $68.14 \pm 1.05$ & $35.35 \pm 3.72$ & $92.87 \pm 0.04$ \\
Genistein & $>100$ & $>100$ & $>100$ & $>100$
\end{tabular}

${ }^{a}$ Each value represents mean $\pm \mathrm{SD}$ of three experiments. aminomethane (Tris) ( $\geq 99 \%)$ was obtained from Solarbio Science \& Technology Co., Ltd. (Beijing, China). Compounds $\mathbf{4 a - 4 d}(\geq 97 \%)$ were synthesized in the laboratory in University of South China.

The stock solution of BSA $(10 \mu \mathrm{M})$ was prepared using $0.05 \mathrm{~mol} \mathrm{~L}^{-1}$ Tris- $\mathrm{HCl}$ buffer solution containing $0.05 \mathrm{~mol} \mathrm{~L}^{-1}$ $\mathrm{NaCl}(\mathrm{pH}=7.4)$. The stock solutions of genistein and its amino acid derivatives $\mathbf{4 a}-\mathbf{4 d}(1000 \mu \mathrm{M})$ were dissolved in methanol. All reactants and solvents used in synthesis were analytically pure and the distilled water was used in experiments.

\subsection{Synthesis of genistein amino acid methyl ester derivatives}

The synthetic route to compounds $\mathbf{4 a - 4 d}$ was shown in Fig. 2. The detailed procedure and characterization spectroscopy of the compounds could be found in the ESI. $\uparrow$

\subsection{Cytotoxicity assays}

2.3.1 Cell culture. All cell lines were purchased from the Cell Bank of Type Culture Collection of Chinese Academy of Sciences (Shanghai, China). MGC-803 was routinely cultured in 

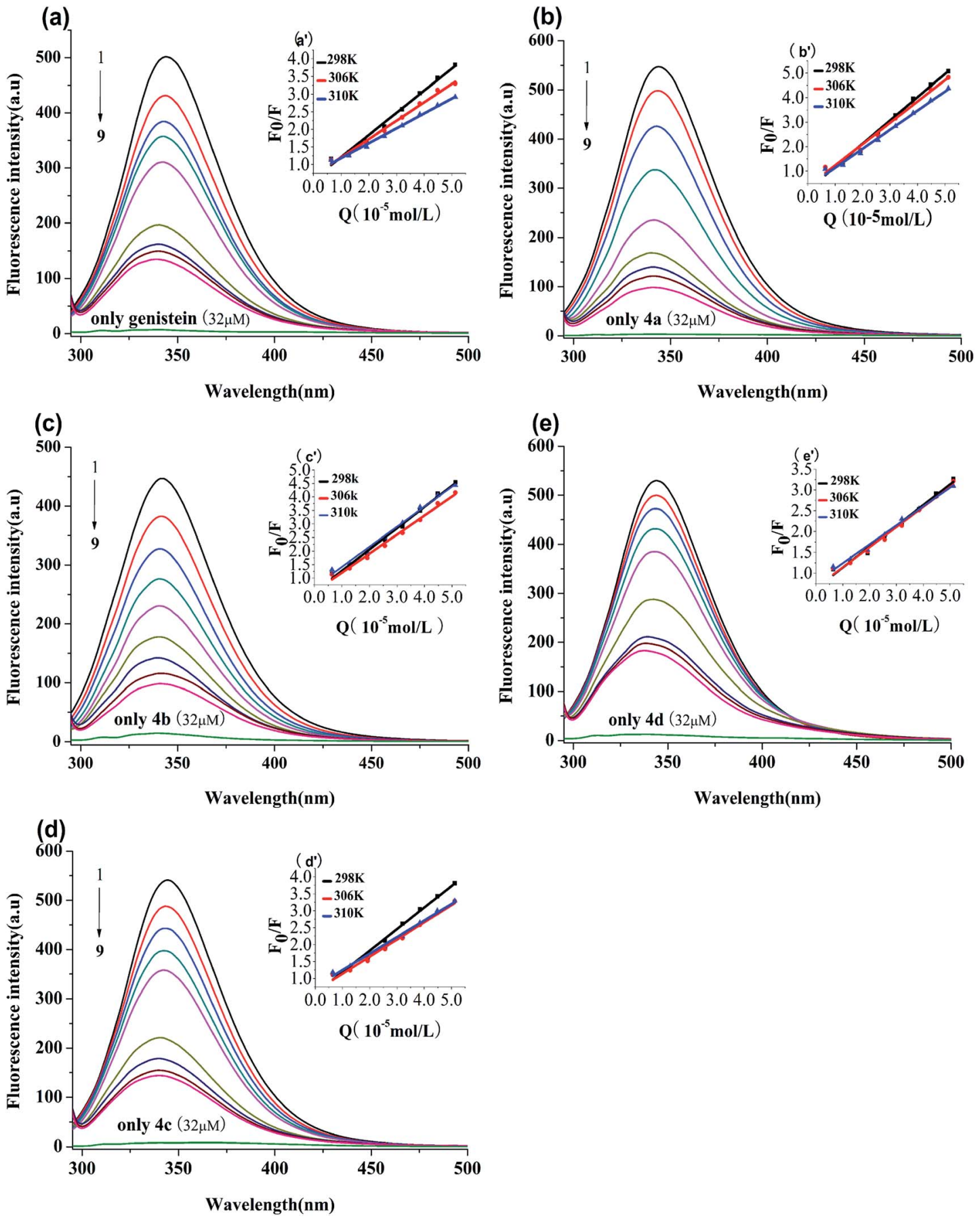

Fig. 3 Fluorescence spectra of BSA (1 $\mu \mathrm{M})$ solution in the presence of genistein (a), 4a (b), 4b (c), 4c (d), 4d (e), at $\lambda_{\text {ex }}=280 \mathrm{~nm}$ at $298 \mathrm{~K}$. The concentrations of all compounds were from 0 to $51.2 \mu \mathrm{M}$ at intervals of $6.4 \mu \mathrm{M}$. Inset: Stern-Volmer plots of genistein $\left(a^{\prime}\right), 4 a\left(b^{\prime}\right), 4 b\left(c^{\prime}\right), 4 c\left(d^{\prime}\right)$, $4 d\left(e^{\prime}\right)$.

RPMI-1640 medium containing 10\% FBS and 1\% penicillin/ streptomycin. MCF-7, HCT-116 and Hela were routinely cultured in medium DMEM/high glucose containing $10 \%$ FBS and $1 \%$ penicillin/streptomycin. All cell lines were incubated in culture at $37{ }^{\circ} \mathrm{C}$ in air with $5 \% \mathrm{CO}_{2}$ for $24 \mathrm{~h}$.

2.3.2 MTT assays. All cell lines were plated in 96-well plates, and each well contains 4000 cells in $100 \mu \mathrm{L}$ culture medium. Then the cells were treated with genistein or its amino acid derivatives dissolved in DMSO at different doses in a $\mathrm{CO}_{2}$ incubator at $37{ }^{\circ} \mathrm{C}$ for $48 \mathrm{~h}$, and DMSO was used as vehicle control. After removing the culture medium, $10 \mu \mathrm{L}$ MTT and 90 $\mu \mathrm{L}$ blank culture medium were added to each well and incubated for additional $4 \mathrm{~h}$ at $37{ }^{\circ} \mathrm{C}$. The absorbance was determined by a microplate-reader at $490 \mathrm{~nm}$.

\subsection{Fluorescence spectra measurements}

2.4.1 Fluorescence quenching spectroscopy. Fluorescence spectra were carried on a F-7000 fluorescence 
spectrophotometer (Japan) fitted out with a $1.0 \mathrm{~cm}$ quartz dish. The excitation wavelength was set at $280 \mathrm{~nm}$ with $5 / 5 \mathrm{~nm}$ slit widths, while the emission wavelength ranged from $290 \mathrm{~nm}$ to $500 \mathrm{~nm}$ at different temperatures. The BSA solution $(1 \mu \mathrm{M})$ was titrated with genistein and its amino acid derivatives $\mathbf{4 a - 4 d}$ for three times. The final concentrations of genistein and $\mathbf{4 a - 4 d}$ were $(0.0,6.4,12.8,19.2,25.6,32,38.4,44.8$, and 51.2) $\mu \mathrm{M}$.

2.4.2 Synchronous fluorescence spectroscopy. The synchronous fluorescence spectra of free BSA and BSAcompounds system were recorded at the emission wavelength from $240 \mathrm{~nm}$ to $400 \mathrm{~nm}$ at $298 \mathrm{~K}$ for three times. The scanning intervals were set to $\Delta \lambda=15 \mathrm{~nm}$ and $\Delta \lambda=60 \mathrm{~nm}\left(\Delta \lambda=\Delta \lambda_{\mathrm{em}}-\right.$ $\Delta \lambda_{\text {ex }}$ ), respectively. Other parameters were the same as Section 2.4.1.

2.4.3 Three-dimensional fluorescence spectroscopy. The 3D fluorescence spectra of genistein and compounds $4 a-4 d$ were recorded with scanning excitation wavelength ranged from $200 \mathrm{~nm}$ to $300 \mathrm{~nm}$ with $10 \mathrm{~nm}$ interval, and emission wavelength ranged from $290 \mathrm{~nm}$ to $470 \mathrm{~nm}$ with $5 \mathrm{~nm}$ interval for three times. Other parameters were the same as Section 2.4.1.

\subsection{Ultraviolet-visible absorption spectroscopy}

The UV spectra were carried on a UV-2450 UV-vis spectrophotometer (Shimadzu, Japan) in the wavelength range of 200$350 \mathrm{~nm}$ at $298 \mathrm{~K}$ for three times. The BSA solutions $(1 \mu \mathrm{M})$ with

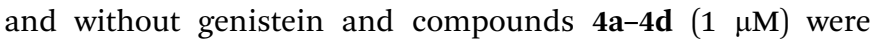
measured. The Tris-HCl buffer solution was used as the blank control.

\subsection{Circular dichroism spectroscopy}

The CD spectra of BSA-genistein and BSA-compounds 4a-4d were performed in the range of 190-250 nm with a scan speed of $100 \mathrm{~nm} \min ^{-1}$ on a J-1500 spectrophotometer (Applied Photophysics Ltd, Surrey, UK) with a $1 \mathrm{~mm}$ quartz dish at $298 \mathrm{~K}$. The concentrations of BSA and compounds $4 \mathbf{a}-\mathbf{4 d}$ were $8 \mu \mathrm{M}$. Spectra were recorded with the resolution of $1 \mathrm{~nm}$, bandwidth of $1 \mathrm{~nm}$ and response of $1 \mathrm{~s}$.

\subsection{Molecular docking}

The X-ray crystal structure of BSA (ID: 4JK4) was got from the Protein Data Bank. The original ligand of the BSA structure was extracted and the water molecule of BSA was removed and hydrogen was added. Atomic charges of BSA were reckoned using Gasteiger-Huckel and AMBER7FF99 method. ${ }^{14}$ Then the 'protomol' file of BSA was generated.

The structures of genistein and its derivatives $\mathbf{4 a - 4 d}$ were optimized by minimization with the minimum RMS of 0.001 using MM2 method implemented by Chem3D Pro 14.0 software. ${ }^{15}$ Then, it was further optimized using Tripos force field and Gasteiger-Huckel methods. Finally, the molecular docking was measured through the Surflex-Dock module in SYBYL-X2.0 software. ${ }^{16}$ The docked conformation was visualized using PyMol. ${ }^{17}$

\section{Results and discussions}

\subsection{Cytotoxic assays}

Genistein and its amino acid derivatives $4 \mathbf{4}-\mathbf{4 d}$ were screened for their cytotoxic activities against four human cancer cell lines (MCF-7, Hela, MGC-803 and HCT-116). The results were summarized in Table 1. As we can see in Table 1, compounds 4a-4d displayed stronger activities than the parent compound genistein, which meant introducing amino acid into genistein was beneficial to improve its anticancer activity. Particularly, compound $\mathbf{4 b}$ bearing alanine chain showed the best cytotoxic activity against MGC-803 cell lines with $\mathrm{IC}_{50}$ value of $12.08 \mu \mathrm{M}$.

\subsection{Fluorescence quenching of BSA}

BSA has endogenous fluorescence, because it possesses tryptophan, tyrosine and phenylalanine residues. ${ }^{18}$ The tryptophan residue is the most important factor in the production of intrinsic fluorescence, which is frequently used as a probe to explore the interaction between drugs and BSA. ${ }^{15}$ In our study, the binding process between genistein, compounds $4 \mathbf{a}-\mathbf{4 d}$ and BSA were investigated via fluorescence spectroscopy at three unequal temperatures. As shown in Fig. 3, BSA had a strong emission peak at around $347 \mathrm{~nm}$ when excited at $280 \mathrm{~nm}$, while the fluorescence absorption of test compounds alone were negligible. With increasing concentrations of genistein and 4a4d (from 0 to $51.2 \mu \mathrm{M}$ ), the fluorescence intensity of BSA decreased dramatically. Besides, a blue shift $(>3 \mathrm{~nm})$ of the maximum emission wavelength $\left(\lambda_{\mathrm{em}}\right)$ appeared, which indicated that genistein and its analogs may bind to BSA, change the micro-environment of tryptophan residue and then quench intrinsic fluorescence of BSA.

\subsection{Quenching mechanism investigation}

3.3.1 Quenching type. The type of fluorescence quenching is usually divided into dynamic and static quenching, which can

Table 2 Quenching constants induced by genistein and compounds $4 \mathrm{a}-4 \mathrm{~d}$ at different temperatures

\begin{tabular}{lllll}
\hline Compounds & $T(\mathrm{~K})$ & $K_{\mathrm{SV}}\left(10^{4} \mathrm{M}^{-1}\right)$ & $K_{\mathrm{q}}\left(10^{12} \mathrm{M}^{-1} \mathrm{~s}^{-1}\right)$ & $r^{2 a}$ \\
\hline Genistein & 298 & $6.2416 \pm 0.0984$ & 6.2416 & 0.9902 \\
& 306 & $5.3472 \pm 0.1242$ & 5.3472 & 0.9911 \\
& 310 & $4.3285 \pm 0.1005$ & 4.3285 & 0.9924 \\
4a & 298 & $9.3690 \pm 0.1055$ & 9.3690 & 0.9900 \\
& 306 & $9.0645 \pm 0.0720$ & 9.0645 & 0.9904 \\
& 310 & $7.7362 \pm 0.1223$ & 7.7362 & 0.9903 \\
4b & 298 & $8.0160 \pm 0.1379$ & 8.0160 & 0.9922 \\
& 306 & $6.9409 \pm 0.0956$ & 6.9409 & 0.9913 \\
& 310 & $7.6019 \pm 0.1604$ & 7.6019 & 0.9924 \\
4c & 298 & $6.2575 \pm 0.1717$ & 6.2575 & 0.9904 \\
& 306 & $5.0010 \pm 0.1067$ & 5.0010 & 0.9906 \\
& 310 & $4.8646 \pm 0.1370$ & 4.8646 & 0.9918 \\
4d & 298 & $4.9339 \pm 0.0500$ & 4.9339 & 0.9896 \\
& 306 & $4.8535 \pm 0.0465$ & 4.8535 & 0.9891 \\
& 310 & $4.5804 \pm 0.0360$ & 4.5804 & 0.9922
\end{tabular}

${ }^{a} r^{2}$ is the correlation coefficient. 
be differentiated from temperature, viscosity and collision rate constant. With the increase of temperature, the static quenching constants decrease owing to the destabilization of the ground-state complex at higher temperature. In contrast, the dynamic quenching constants increase with the rise of temperature, because the molecular diffusion coefficient becomes larger. ${ }^{15}$

The Stern-Volmer equation is often applied to reveal the fluorescence quenching mechanism: ${ }^{19}$

$$
\frac{F_{0}}{F}=1+K_{\mathrm{SV}}[\mathrm{Q}]=1+K_{\mathrm{q}} \tau_{0}[\mathrm{Q}]
$$

where $F_{0}$ and $F$ are the fluorescence intensities of complex with and without of quencher, respectively. ${ }^{18}$ [Q] denotes the concentration of quencher. $K_{\mathrm{sv}}$ and $K_{\mathrm{q}}$ are quenching constant and quenching rate constant of BSA, respectively. ${ }^{20} \tau_{0}$ is the lifetime of fluorophore in the absence of genistein, $4 \mathbf{a}-\mathbf{4 d}$ and equals to $10^{-8} \mathrm{S.}^{21}$ The inset of Fig. 3 showed the Stern-Volmer plots of compounds-BSA system, which displayed good linear
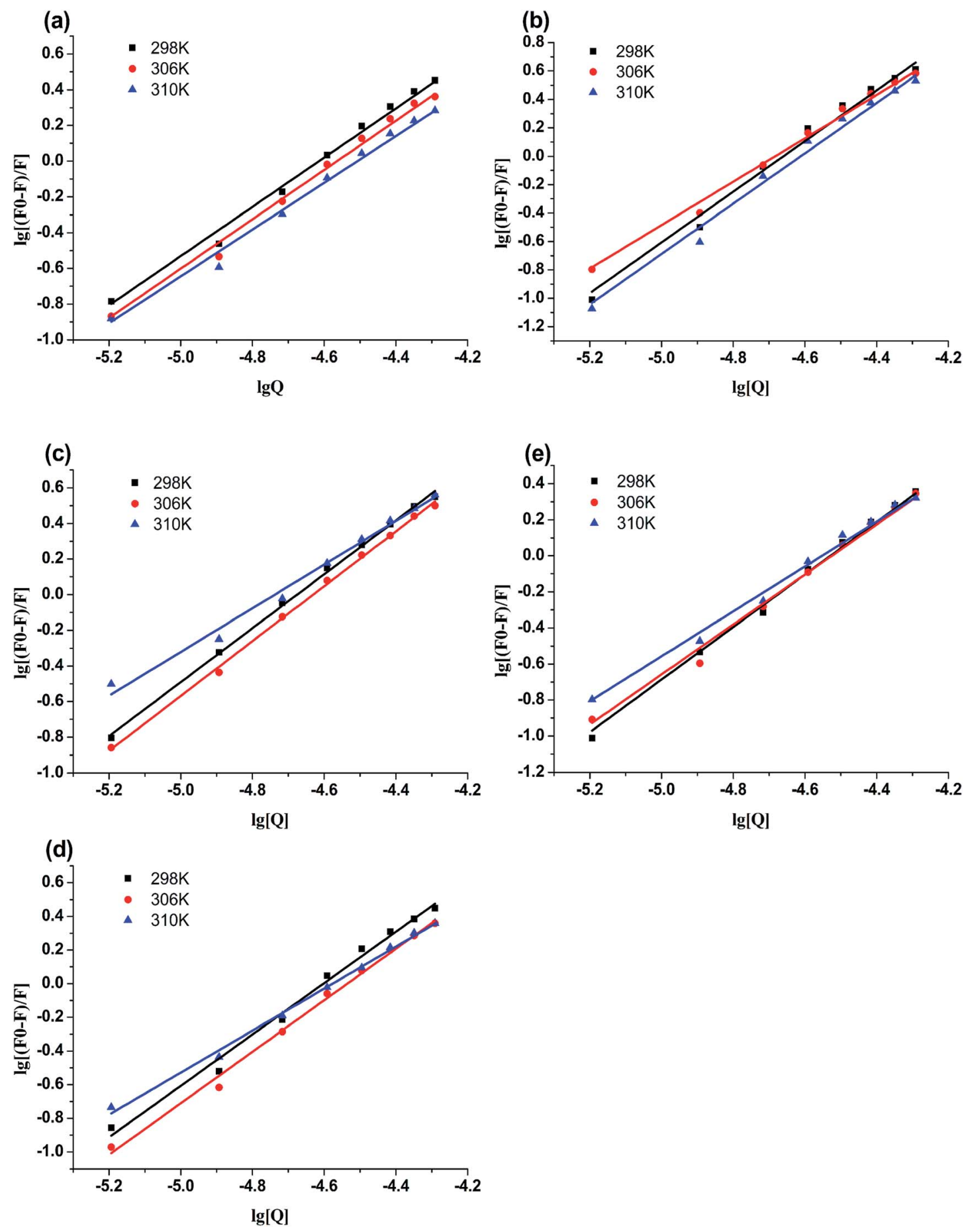

Fig. 4 Double-log plots of genistein (a), 4a (b), 4b (c), 4c (d) or 4d (e) binding to BSA at different temperatures. 
relationship. It can be observed that the $K_{\mathrm{sv}}$ values decreased gradually with rise in temperature in Table 2 . Furthermore, $K_{\mathrm{q}}$ values were in the order of $10^{12}$, which were larger than the maximum diffusion collisional rate constant $\left(2.0 \times 10^{10} \mathrm{M}^{-1}\right.$ $\left.\mathrm{s}^{-1}\right) .{ }^{22}$ Hence, we propose that the quenching mechanism of BSA fluorescence was a ground-state complex formed rather than dynamic collision.

3.3.2 Binding parameters. Double-logarithmic eqn (2) is often used to explore the binding parameters of static quenching, such as binding constant $\left(K_{\mathrm{b}}\right)$ and binding sites $(n):^{23}$

$$
\log \frac{F_{0}-F}{F_{0}}=\log K_{\mathrm{b}}+n \log [\mathrm{Q}]
$$

The plots of $\log \left[\left(F_{0}-F\right) / F_{0}\right]$ versus $\log [\mathrm{Q}]$ were displayed in Fig. 4. The values of $K_{\mathrm{b}}$ and $n$ for compounds-BSA system were recorded in Table 3. According to Table 3, all the number of binding sites were more than 1 , which meant there was not only a strongly single binding site between compounds $\mathbf{4 a - 4 d}$ and BSA. ${ }^{24}$ The binding constants $\left(K_{\mathrm{b}}\right)$ decreased with rise in temperature, which were consistent with the trends of $K_{\mathrm{sv}}$. Furthermore, the binding constants of compounds-BSA increased in the following order: $4 \mathbf{a}>\mathbf{4 b}>\mathbf{4 c}>\mathbf{4 d}>$ genistein, which meant the introduction of amino acid into genistein can enhance its interaction with BSA. And the larger the alkyl chain of amino acid, the lower affinity to BSA. The reasons for this phenomenon are as follows: introducing amino acid into genistein can enhance its hydrophobicity, which was beneficial to penetrate into the hydrophobic tryptophan residues of BSA. Besides, the amide group of compounds 4a-4d can form N-H-O or O-H-S or N-H-S type of hydrogen bonds with amino groups, hydroxyl groups and sulfhydryl groups on the surface of BSA. On the other side, with the increase of the length of the alkyl chain of compounds 4a-4d, the effects of steric hindrance became stronger, which weakened their capacities to bind with BSA.
3.3.3 Binding forces to BSA. To clarify the binding forces between genistein, $\mathbf{4 a - 4 d}$ and BSA, the thermodynamic parameters were studied via the van't Hoff equations: ${ }^{25}$

$$
\begin{gathered}
\ln K=-\frac{\Delta H}{R T}+\frac{\Delta S}{R} \\
\Delta G=-R T \ln K=\Delta H-T \Delta S
\end{gathered}
$$

where $T$ denotes the experimental temperature, $K$ denotes the binding constant at the corresponding temperature, $R$ is the gas constant. ${ }^{26}$ According to Ross and Subramanian's previous research, ${ }^{27}$ the characteristic for van der Waals interactions or/ and hydrogen bonding is $\Delta H<0$ and $\Delta S<0$, for hydrophobic interaction is $\Delta H>0$ and $\Delta S>0$, and for electrostatic interactions is $\Delta H \approx 0$ and $\Delta S>0 .{ }^{28}$ The calculation results of the thermodynamic parameters were recorded in Table 3. As shown in Table 3, the $\Delta G<0$ meant that the interactions between genistein, compounds $\mathbf{4 a - 4 d}$ and BSA were spontaneous. The $\Delta H<0$ and $\Delta S<0$ suggested that van der Waals or hydrogen bonding was the main force in the process of binding compounds $\mathbf{4 a - 4 d}$ to BSA.

\subsection{Conformation investigation}

3.4.1 Synchronous fluorescence spectroscopy. The microenvironment of Trp and Tyr residues of BSA is often studied by synchronous fluorescence spectroscopy. When the intervals of wavelength of synchronous fluorescence spectra are equal to $15 \mathrm{~nm}$ and $60 \mathrm{~nm}$, which are characteristics of Tyr and Trp residues, respectively. ${ }^{29}$ Generally, the shift of maximum emission wavelength is used to represent the change of the polarity of the micro-environment surrounding Tyr or Trp residues. ${ }^{30}$ The red shift suggests that the polarity surrounding Tyr or Trp residues increases and hydrophobicity decreases. In reverse, the blue shift means the hydrophobicity increases and the polarity decreases. ${ }^{31}$ The synchronous fluorescence spectra of

\begin{tabular}{|c|c|c|c|c|c|c|c|c|}
\hline Compounds & $T(\mathrm{~K})$ & $\log K_{\mathrm{b}}$ & $K_{\mathrm{b}}\left(\mathrm{M}^{-1}\right)$ & $n$ & $R^{2 a}$ & $\Delta H\left(\mathrm{~kJ} \mathrm{M}^{-1}\right)$ & $\Delta S\left(\mathrm{~J} \mathrm{~K}^{-1} \mathrm{M}^{-1}\right)$ & $\Delta G_{\exp }^{b}\left(\mathrm{~kJ} \mathrm{M}^{-1}\right)$ \\
\hline \multirow[t]{3}{*}{ Genistein } & 298 & $6.58 \pm 0.13$ & $3.90 \times 10^{6}$ & 1.42 & 0.9933 & \multirow[t]{3}{*}{-59.09} & \multirow[t]{3}{*}{-71.83} & -37.69 \\
\hline & 306 & $6.36 \pm 0.16$ & $2.41 \times 10^{6}$ & 1.39 & 0.9930 & & & -37.11 \\
\hline & 310 & $6.17 \pm 0.05$ & $1.49 \times 10^{6}$ & 1.37 & 0.9920 & & & -36.83 \\
\hline \multirow[t]{3}{*}{$4 a$} & 298 & $8.64 \pm 0.04$ & $4.35 \times 10^{8}$ & 1.86 & 0.9909 & \multirow[t]{3}{*}{-123.52} & \multirow[t]{3}{*}{-249.37} & -49.21 \\
\hline & 306 & $8.02 \pm 0.06$ & $1.05 \times 10^{8}$ & 1.72 & 0.9923 & & & -47.21 \\
\hline & 310 & $7.81 \pm 0.08$ & $6.52 \times 10^{7}$ & 1.66 & 0.9904 & & & -46.22 \\
\hline \multirow[t]{3}{*}{$4 b$} & 298 & $7.17 \pm 0.14$ & $1.54 \times 10^{7}$ & 1.86 & 0.9909 & \multirow[t]{3}{*}{-175.21} & \multirow[t]{3}{*}{-446.98} & -42.01 \\
\hline & 306 & $7.10 \pm 0.03$ & $1.26 \times 10^{7}$ & 1.53 & 0.9980 & & & -38.43 \\
\hline & 310 & $5.81 \pm 0.08$ & $6.46 \times 10^{5}$ & 1.23 & 0.9896 & & & -36.65 \\
\hline \multirow[t]{3}{*}{$4 \mathrm{c}$} & 298 & $7.07 \pm 0.10$ & $1.19 \times 10^{7}$ & 1.54 & 0.9919 & \multirow[t]{3}{*}{-177.34} & \multirow[t]{3}{*}{-456.55} & -41.29 \\
\hline & 306 & $6.92 \pm 0.03$ & $8.38 \times 10^{6}$ & 1.53 & 0.9947 & & & -37.63 \\
\hline & 310 & $5.69 \pm 0.05$ & $4.97 \times 10^{5}$ & 1.24 & 0.9958 & & & -35.81 \\
\hline \multirow[t]{3}{*}{ 4d } & 298 & $6.80 \pm 0.19$ & $6.65 \times 10^{6}$ & 1.50 & 0.9977 & \multirow[t]{3}{*}{-126.67} & \multirow[t]{3}{*}{-292.42} & -39.53 \\
\hline & 306 & $6.66 \pm 0.15$ & $4.78 \times 10^{6}$ & 1.47 & 0.9958 & & & -37.19 \\
\hline & 310 & $5.84 \pm 0.07$ & $7.04 \times 10^{5}$ & 1.28 & 0.9937 & & & -36.02 \\
\hline
\end{tabular}
compounds-BSA are exhibited in Fig. 5. As shown in Fig. 5, it

Table 3 The binding and thermodynamic parameters for genistein and its derivatives binding to BSA

${ }^{a} R^{2}$ is the correlation coefficient. ${ }^{b} \Delta G_{\exp }=\Delta H-T \Delta S$. 
was apparent that the fluorescence intensities of Trp and Try residues both decreased along with a red shift of the emission peak, which indicated that the hydrophobicity around Trp and Tyr residues decreased. Moreover, the level of red shift for Typ residues $(>4.0 \mathrm{~nm})$ was greater compared to that for $\operatorname{Trp}$ residues $(>2.5 \mathrm{~nm})$, indicating that the micro-environment around Typ residues became less hydrophobic.

3.4.2 UV-vis absorption spectroscopy. The conformational change of protein caused by binding to the ligand is usually studied by UV-vis spectroscopy. In order to verify the
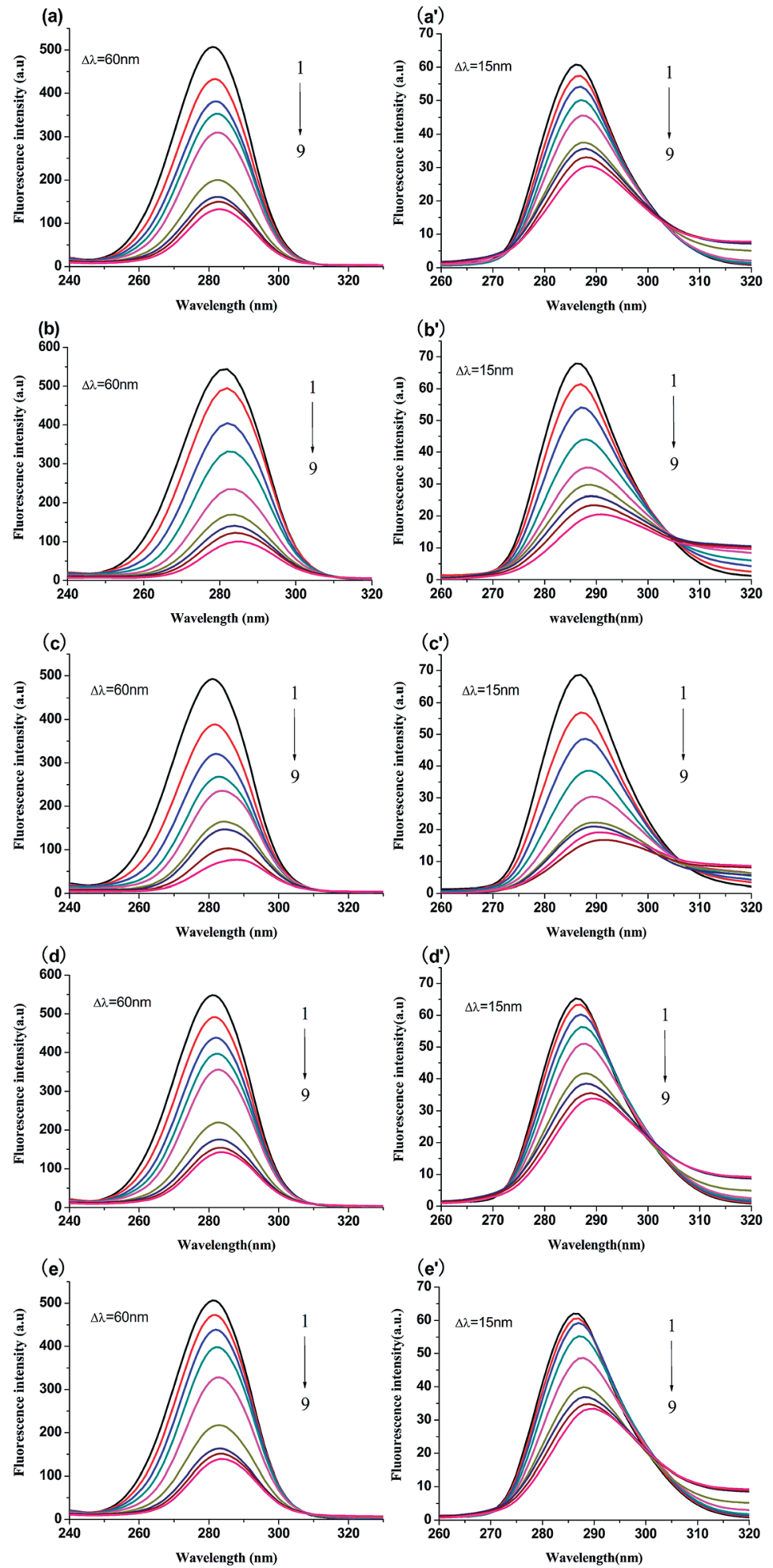

Fig. 5 Synchronous fluorescence spectra of BSA $(1 \mu \mathrm{M})$ with and without genistein, $4 \mathrm{a}, 4 \mathrm{~b}, 4 \mathrm{c}$ or $4 \mathrm{~d}$ at $298 \mathrm{~K}$ when $\Delta \lambda=60 \mathrm{~nm}(\mathrm{a}-\mathrm{e})$ and $\Delta \lambda=$ $15 \mathrm{~nm}\left(\mathrm{a}^{\prime}-\mathrm{e}^{\prime}\right)$. The concentrations of all compounds from 0 to $51.2 \mu \mathrm{M}$ at intervals of $6.4 \mu \mathrm{M}$. 
conformational change, the UV-absorption spectra of free BSA and BSA-compounds system are implemented. As shown in Fig. 6, BSA has two absorption peaks: the strong peak around $208 \mathrm{~nm}$ reflected the framework of protein and the peak around $280 \mathrm{~nm}$ ascribed to absorption of Trp, Tyr, and Phe residues. ${ }^{32}$ The absorbance of the strong peak decreased with addition of genistein and 4a-4d along with a red shift, showing that BSAcompounds complex were formed. They could reduce the $\alpha$ helical content and change the conformation of BSA. ${ }^{33}$
3.4.3 3D fluorescence spectra. Three-dimensional (3D) fluorescence spectrum can show us more intuitive information about the micro-environmental changes of endogenous fluorophores (Trp, Typ, Phe) and conformational changes of proteins when binding to the ligands. The 3D fluorescence spectrum of BSA and BSA-compounds system were showed in Fig. 7 and the data were summarized in Table 4. As we can see, three peaks were observed in the $3 \mathrm{D}$ fluorescence spectra. Peak a $\left(\lambda_{\text {ex }}=\lambda_{\text {em }}\right)$ was the Rayleigh scattering peak and peak 1
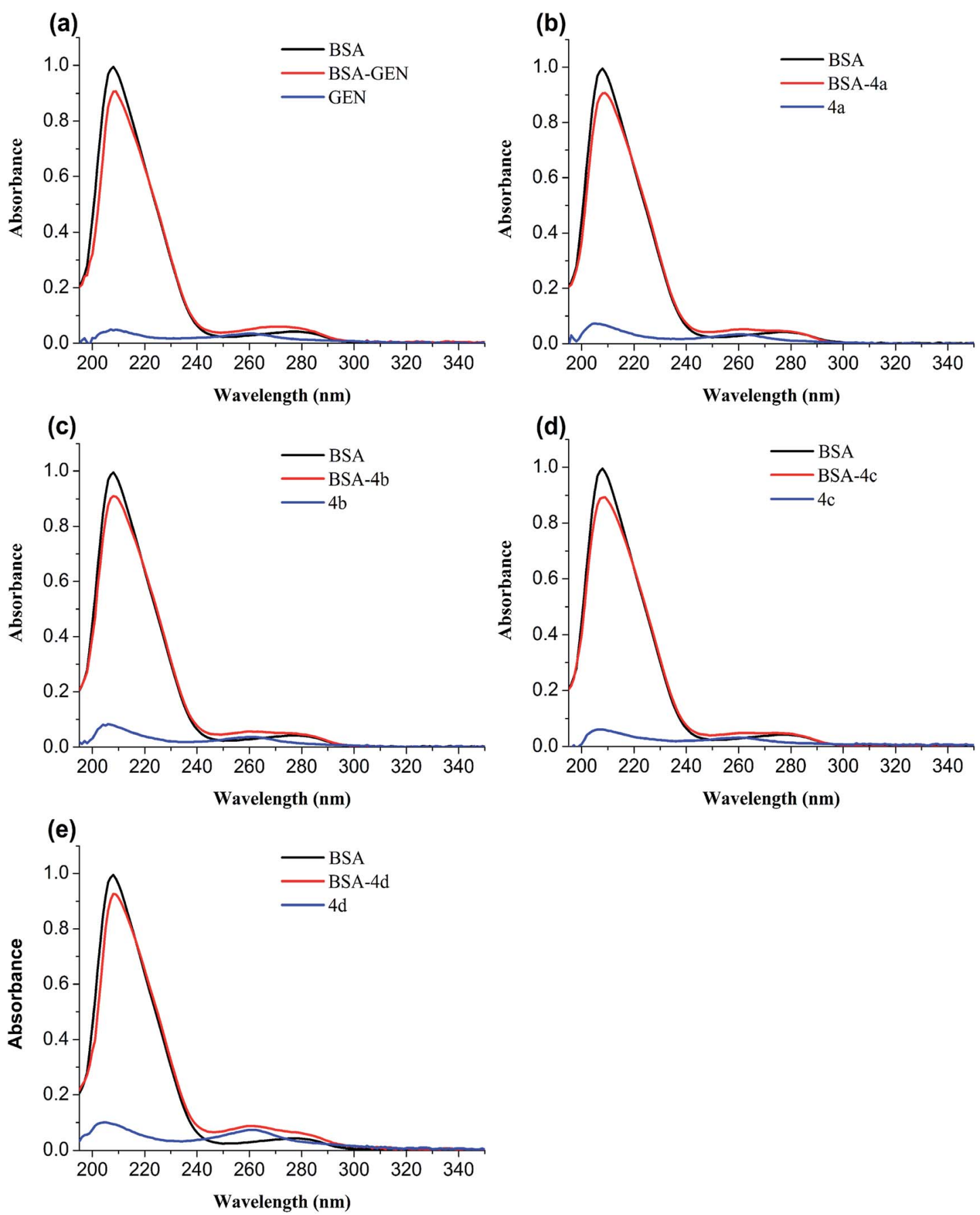

Fig. 6 UV absorption spectra of BSA in the absence and presence of genistein (a), 4a (b), 4b (c), 4c (d), or 4d (e). The concentrations of BSA and compounds $4 a-4 d$ were $1 \mu \mathrm{M}$. 
corresponded to the characteristic peak of polypeptide backbone structure due to $\pi \rightarrow \pi^{*}$ transition, while peak 2 represented the spectral feature of tryptophan and tyrosine residues in protein. ${ }^{25,34,35}$ As shown in Fig. 7, with the addition of genistein and compounds $\mathbf{4 a - 4 d}$, the intensity of emission peak a increased, which indicating the diameter of BSA increased. In contrast, the fluorescence intensities of emission peak 1 and emission peak 2 decreased dramatically along with a slight red shift ( $\sim 2 \mathrm{~nm}$ ) of peak 2, revealing that the conformation of the peptide backbone was changed and the polarity surrounding endogenous fluorophore (Trp and Typ residues) of BSA increased. Detailed data were recorded in Table 4.

3.4.4 Circular dichroism spectroscopy. CD spectra are commonly applied to study the secondary structure changes in protein during ligand-protein binding. In our study, the CD spectra of BSA with and without compounds 4a-4d were
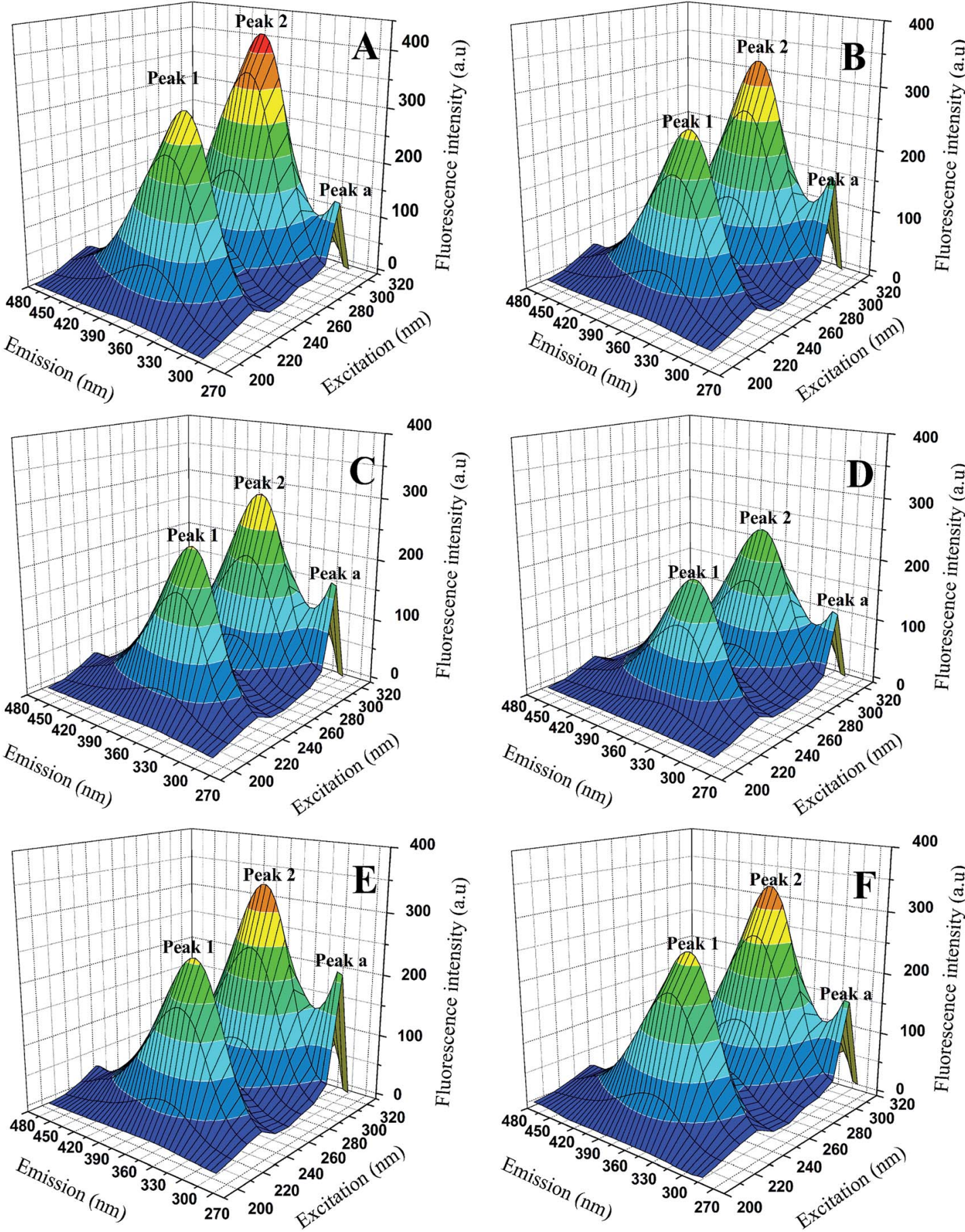

Fig. 7 3D fluorescence spectra of BSA (A) and BSA-genistein (B), BSA-4a (C), BSA-4b (D), BSA-4c (E), BSA-4d (F). $C_{(\mathrm{compounds})}=3.0 \mu \mathrm{M}, \mathrm{C}_{(\mathrm{BSA})}=$ $1.0 \mu \mathrm{M}$. 
Table 4 3D fluorescence spectral parameters of BSA with and without genistein or $4 a-4 d$ at $298 \mathrm{~K}$

\begin{tabular}{llll}
\hline System & Peak no. & $\begin{array}{l}\text { Position } \lambda_{\mathrm{ex}} / \lambda_{\mathrm{em}} \\
(\mathrm{nm} / \mathrm{nm})\end{array}$ & $\begin{array}{l}\text { Intensity } \\
(\mathrm{a} . \mathrm{u})\end{array}$ \\
\hline$[\mathrm{BSA}]$ & 1 & $230 / 355$ & 326.1 \\
& 2 & $280 / 353$ & 422.7 \\
& $\mathrm{a}$ & $290 / 295$ & 138.0 \\
{$[\mathrm{BSA}]:[\mathbf{4 a}]=1: 3$} & 1 & $230 / 355$ & 249.8 \\
& 2 & $280 / 355$ & 300.8 \\
& $\mathrm{a}$ & $290 / 295$ & 167.9 \\
{$[\mathrm{BSA}]:[\mathbf{4 b}]=1: 3$} & 1 & $230 / 355$ & 199.8 \\
& 2 & $280 / 355$ & 242.6 \\
& $\mathrm{a}$ & $290 / 295$ & 140.7 \\
{$[\mathrm{BSA}]:[\mathbf{4 c}]=1: 3$} & 1 & $230 / 355$ & 254.6 \\
& 2 & $280 / 355$ & 338.3 \\
{$[\mathrm{BSA}]:[\mathbf{4 d}]=1: 3$} & $\mathrm{a}$ & $290 / 295$ & 210.9 \\
& 1 & $230 / 355$ & 263.8 \\
& 2 & $280 / 355$ & 331.4 \\
{$[\mathrm{BSA}]:[$ genistein] $=1: 3$} & 1 & $290 / 295$ & 155.5 \\
& 2 & $230 / 355$ & 259.2 \\
& $\mathrm{a}$ & $280 / 355$ & 333.2 \\
& $\mathrm{a}$ & $290 / 295$ & 159.6 \\
\hline
\end{tabular}

measured and shown in Fig. 8. Obviously, there were two negative absorption bands at nearby $208 \mathrm{~nm}$ and $222 \mathrm{~nm}$, respectively. These are the characteristic peaks of the $\alpha$-helical of protein. ${ }^{36}$ As shown in Fig. 8, with the compounds added, the absorption intensity of $\alpha$-helical characteristic peak decreased, which meant that the combination of compounds 4a-4d with BSA could decrease the content of $\alpha$-helical. This result was in accordance with the results of UV and synchronous fluorescence experiments. From Fig. 8, we can also find out that the changes of the content of $\alpha$-helical of protein induced by compounds $\mathbf{4 a - 4 d}$ were greater than the parent skeleton genistein, which further confirmed that introducing amino acid group into genistein can enhance its interaction with BSA.

\subsection{Molecular docking}

Molecular docking has become a popular method to predict the interaction between ligands and proteins. The conformations of BSA-compounds complexes with the lowest energy were represented in Fig. 9. Obviously, genistein and compounds $\mathbf{4 a - 4 c}$ inserted into the sub-domain IIA of BSA, while compound $\mathbf{4 d}$ was apt to bind on site IIIA. There were hydrogen bondings interactions of genistein and compounds 4a-4d with some amino acid residues in the binding pocket. For instance, genistein formed three hydrogen bondings with Arg208, Leu326 and Leu480 and compound 4c formed three hydrogen bondings with Tyr160, Asp111 and Glu125. The binding pockets formed by the interactions of genistein and compounds 4a-4d with BSA were close to Trp213 residue, and this was the reason for the fluorescence quenching of these ligands binding to BSA. Besides, the distance between compounds $\mathbf{4 a - 4 b}$ and Trp 213 was closer compared to genistein and $\mathbf{4 c - 4 d , ~ w h i c h ~ i n d i c a t i n g ~ t h a t ~ t h e ~ a b i l i t i e s ~ o f ~} \mathbf{4 a - 4 b}$ to quench the fluorescence of BSA were greater than that of genistein and $\mathbf{4 c - 4 d}$. The amide bond and ester groups of $\mathbf{4 a -}$

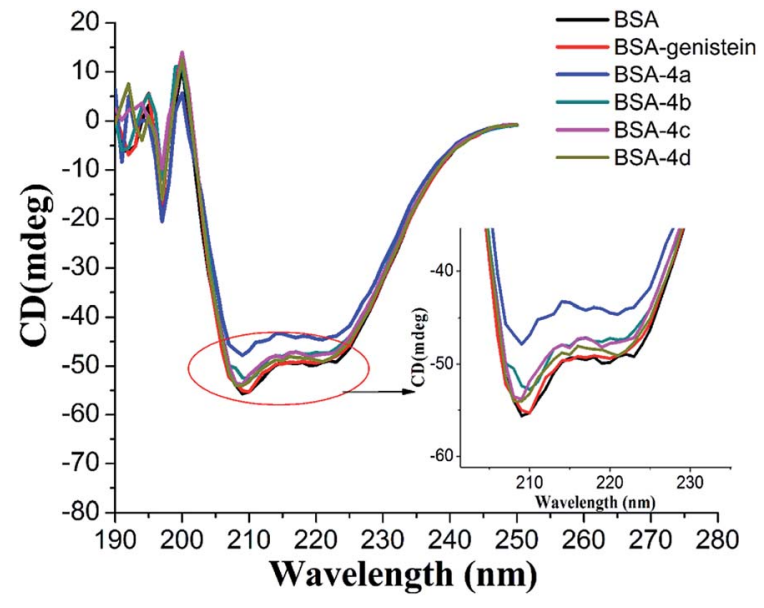

Fig. 8 CD spectra of BSA with and without genistein, $4 a, 4 b, 4 c$, and $4 \mathrm{~d}$. The concentrations of BSA and all compounds were $4 \mu \mathrm{M}$.

4d inserted into hydrophobic loop created by Arg198, Ser201, Ala209, Phe205, Leu210 residues, indicating that van der Waals force was also involved. Furthermore, there were many hydrophobic residues surrounding genistein and compounds 4a-4d, which suggested that hydrophobic interactions may exist. For example, compound $\mathbf{4 b}$ was surrounded by Leu197, Ala209, Ala212, Leu210, Leu326, Trp213, Gly327, Lys350 residues. From the above analyses, it can be speculated that the priority driving force of the interaction between genistein, compounds $\mathbf{4 a - 4 d}$ and BSA was hydrogen bonding, and van der Waals force and hydrophobic interaction played a minor part, which was consistent with the results of Section 3.3.3.

\section{Conclusion}

Herein, four novel genistein amino acid derivatives $4 \mathbf{4}-\mathbf{4 d}$ were synthesized and their cytotoxicity activities were evaluated. The results showed that compound $\mathbf{4 b}$ with alanine chain showed best activity against MGC-803 cell lines with $\mathrm{IC}_{50}$ value of 12.08 $\mu \mathrm{M}$. Then the interactions between compounds 4a-4d and BSA were explored by spectral techniques and molecular docking. Fluorescence quenching experiments proved that all five compounds genistein, $\mathbf{4 a}-\mathbf{4 d}$ quenched the fluorescence of BSA through static quenching mechanism. The thermodynamic data suggested that hydrogen bonding interaction played a major role in the bonding process, van der Waals and hydrophobic force were also involved. Synchronous and 3D fluorescence spectra indicated combination of compounds and BSA could change the microenvironment of endogenous fluorophore (Trp and Typ). The CD spectra demonstrated the content of $\alpha$-helical in the BSA secondary structure was obviously decreased in the presence of compounds 4a-4d. In conclusion, introducing the amino acid group into genistein can not only improve its antitumor activity, but also enhance its binding affinity with BSA. These data were of great significance for future studies on the structural modification and pharmacokinetics of genistein in vivo. 
A

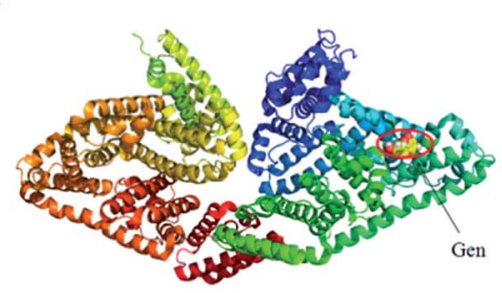

B

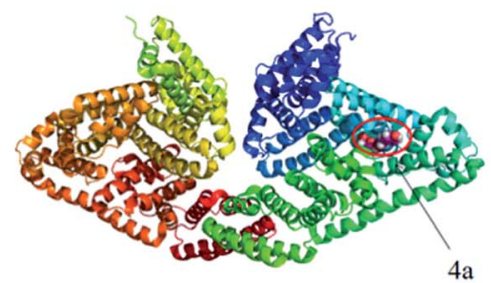

C

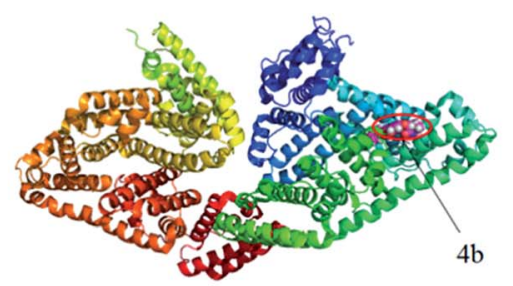

D

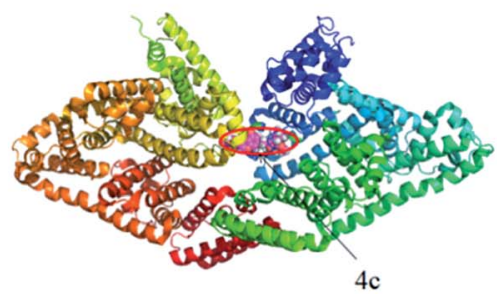

E

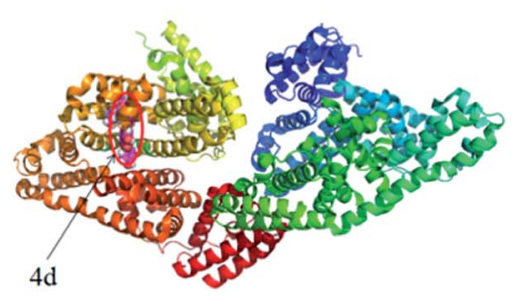

$\mathrm{A}^{\prime}$

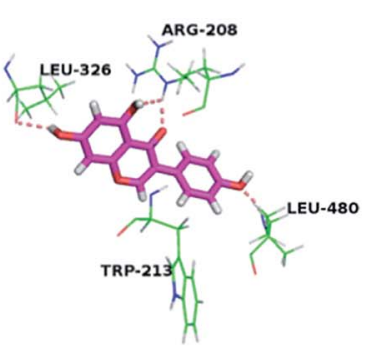

B'

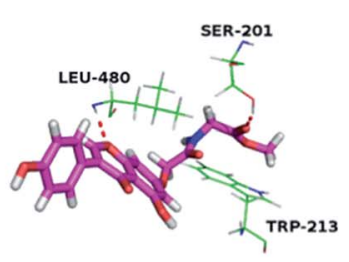

$\mathrm{C}^{\prime}$

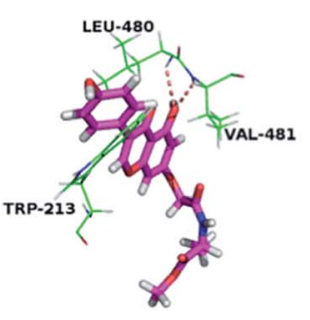

D'

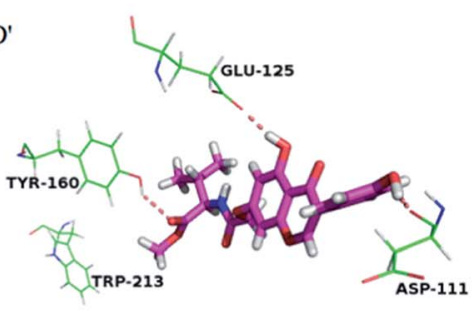

$E^{\prime}$

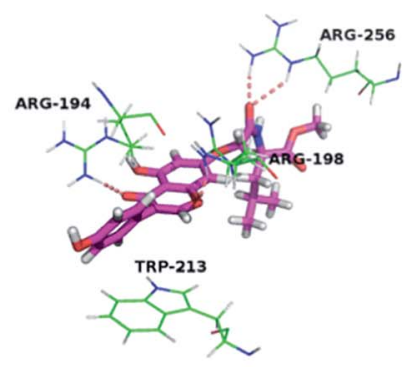

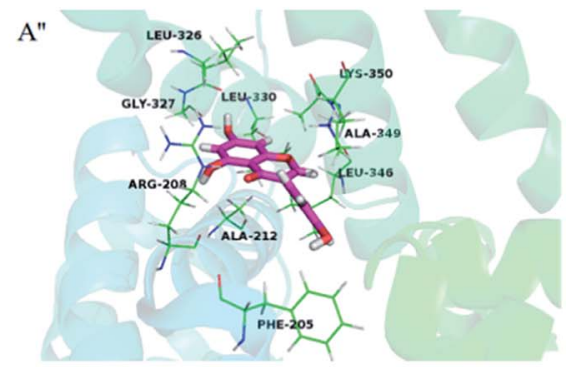
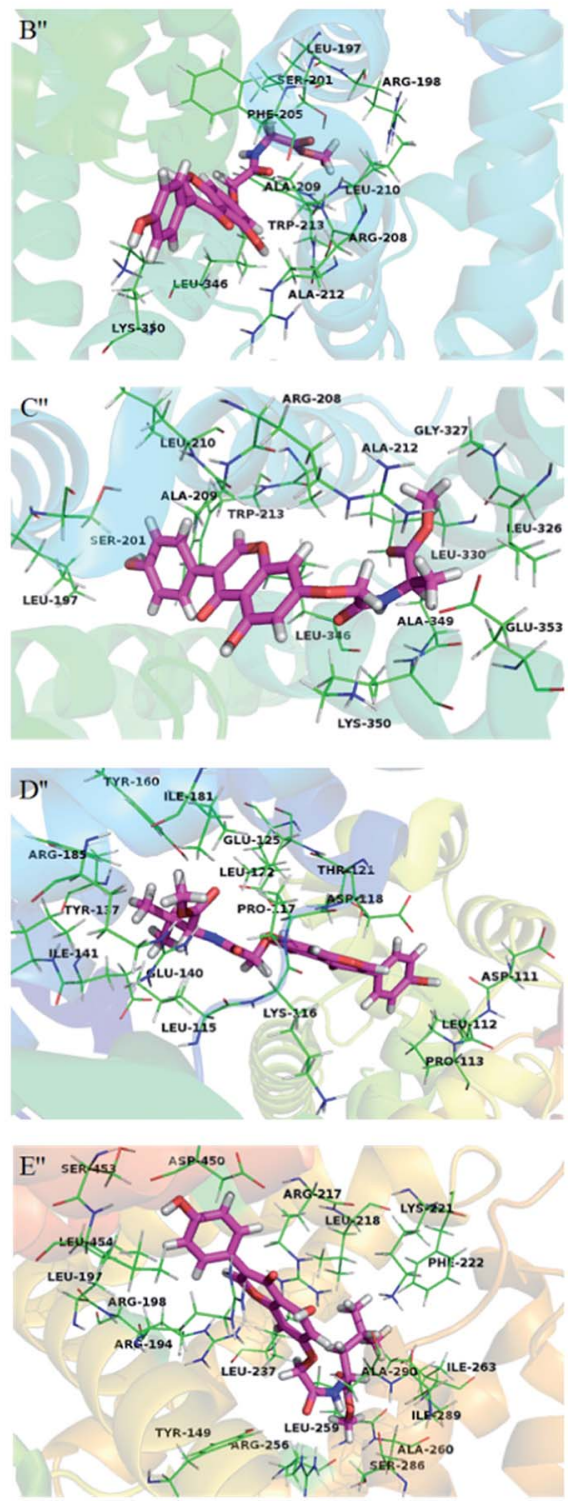

Fig. 9 The lowest energy conformation of compounds-BSA complexes obtained from molecular docking (A-E). The hydrogen bonding interactions of genistein and compounds $4 a-4 d$ with amino acid residues of BSA $\left(A^{\prime}-E^{\prime}\right)$. Amino acid residues surrounding genistein and compounds $4 a-4 d$ within $3 \AA$ ( $\left.A^{\prime \prime}-E^{\prime \prime}\right)$. Notes: labels of A-E correspond to genistein and compounds $4 a-4 d$, respectively.

\section{Conflicts of interest}

There are no conflicts to declare.

\section{Acknowledgements}

This work was supported by the Construct Program of the Key Discipline in Hunan Province, and Key and Youth Project of Hunan Provincial Education Department of China (17A190 and 17B234). 


\section{References}

1 Å. FrostellKarlsson, A. Remaeus, H. Roos, K. Andersson, P. Borg, A. Markku Hämäläinen and R. Karlsson, J. Med. Chem., 2000, 43, 1986-1992.

2 S. Pal and C. Saha, J. Biomol. Struct. Dyn., 2014, 32, 11321147.

3 Z. Tian, F. Zang, W. Luo, Z. Zhao, Y. Wang, X. Xu and C. Wang, J. Photochem. Photobiol., B, 2015, 142, 103-109.

4 D. C. Carter, B. Chang, J. X. Ho, K. Keeling and Z. Krishnasami, FEBS J., 1994, 226, 1049-1052.

5 Y. Y. Lou, K. L. Zhou, J. H. Shi and D. Q. Pan, J. Photochem. Photobiol., B, 2017, 173, 589-597.

6 H. Hong, M. R. Landauer, M. A. Foriska and G. D. Ledney, J. Basic Microbiol., 2010, 46, 329-335.

7 E. T. Menze, A. Esmat, M. G. Tadros, A. B. Abdelnaim and A. E. Khalifa, PLoS One, 2015, 10, e0117223.

8 C. Rowell, D. M. Carpenter and C. A. Lamartiniere, J. Nutr., 2005, 135, 2953S-2959S.

9 S. F. Wang, Q. Jiang, Y. H. Ye, Y. Li and R. X. Tan, Bioorg. Med. Chem., 2005, 13, 4880-4890.

10 M. Krecmerova, Mini-Rev. Med. Chem., 2017, 17, 818-833.

11 B. S. Vig, K. M. Huttunen, K. Laine and J. Rautio, Adv. Drug Delivery Rev., 2013, 65, 1370-1385.

12 J. Xiong, H. F. Zhu, Y. J. Zhao, Y. J. Lan, J. W. Jiang, J. J. Yang and S. F. Zhang, Molecules, 2009, 14, 3142-3152.

13 J. Zhang, X. J. Wang, Y. J. Yan and W. S. Xiang, J. Agric. Food Chem., 2011, 59, 7506-7513.

14 Y. Guo, L. Shen, X. Yao, Y. Liu, Y. Liu, H. Chen, K. Min and X. Zheng, Luminescence, 2017, 32, 1368-1384.

15 Y. Y. Lou, K. L. Zhou, D. Q. Pan, J. L. Shen and J. H. Shi, J. Photochem. Photobiol., B, 2017, 167, 158-167.

16 A. K. Ghose, E. P. Jaeger, P. J. Kowalczyk, M. L. Peterson and A. M. Treasurywala, J. Comput. Chem., 1993, 14, 1050-1065.

17 D. Seeliger and B. L. D. Groot, J. Comput.-Aided Mater. Des., 2010, 24, 417-422.

18 X. Tang, P. Tang and L. Liu, Molecules, 2017, 22, pii: E1036.

19 R. Ma, H. Pan, T. Shen, P. Li, Y. Chen, Z. Li, X. Di and S. Wang, Molecules, 2017, 22, pii: E1317.
20 N. Barbero, E. Barni, C. Barolo, P. Quagliotto, G. Viscardi, L. Napione, S. Pavan and F. Bussolino, Dyes Pigm., 2009, 80, 307-313.

21 J. H. Shi, W. Qi, D. Q. Pan, T. T. Liu and J. Min, J. Biomol. Struct. Dyn., 2016, 35, 1529-1546.

22 R. A. Singha, D. R. Tripathy, A. Chatterjee and S. Dasgupta, Spectrochim. Acta, Part A, 2013, 102, 393-402.

23 B. Sandhya, A. H. Hegde, S. S. Kalanur, U. Katrahalli and J. Seetharamappa, J. Pharm. Biomed. Anal., 2011, 54, 11801186.

24 J. Q. Tong, F. F. Tian, Y. Liu and F. L. Jiang, RSC Adv., 2014, 4, 59686-59696.

25 T. Ren, L. Zhang, J. Wang, C. Song, R. Wang and J. Chang, J. Biomol. Struct. Dyn., 2016, 35, 1-12.

26 A. N. Nasruddin, S. R. Feroz, A. K. Mukarram, S. B. Mohamad and S. Tayyab, J. Lumin., 2016, 174, 77-84.

27 P. D. Ross and S. Subramanian, Biochemistry, 1981, 20, 30963102.

28 G. F. Shen, T. T. Liu, Q. Wang, M. Jiang and J. H. Shi, J. Photochem. Photobiol., B, 2015, 153, 380-390.

29 B. Hemmateenejad, M. Shamsipur, F. Samari, T. Khayamian, M. Ebrahimi and Z. Rezaei, J. Pharm. Biomed. Anal., 2012, 67-68, 201-208.

30 J. H. Shi, W. Qi, D. Q. Pan, T. T. Liu and J. Min, J. Biomol. Struct. Dyn., 2016, 35, 1529-1546.

31 J. H. Shi, Y. Y. Zhu, J. Wang and J. Chen, Luminescence, 2015, 30, 44-52.

32 E. L. Gelamo, C. H. Silva, H. Imasato and M. Tabak, Biochim. Biophys. Acta, Biomembr., 2002, 1594, 84-99.

33 J. H. Shi, D. Q. Pan, M. Jiang, T. T. Liu and Q. Wang, J. Biomol. Struct. Dyn., 2016, 35, 2211-2223.

34 M. S. Ali, M. Altaf and H. A. Al-Lohedan, J. Photochem. Photobiol., B, 2017, 173, 108-119.

35 D. Li, Y. Wang, J. Chen and B. Ji, Spectrochim. Acta, Part A, 2011, 79, 680-686.

36 Z. X. Chi, R. T. Liu, Y. Teng, X. Y. Fang and C. Z. Gao, J. Agric. Food Chem., 2010, 58, 10262-10269. 